\title{
Antibiotic resistance is a common problem in medicine and veterinary
}

\author{
Michael Naumov 1,*, Ludmila Reznichenko², Yana Masalykina ${ }^{3}$ and Ivan Styazhkin ${ }^{4}$ \\ ${ }^{1}$ Kursk State Agricultural Academy, Russia \\ 2 Belgorod State Agricultural University, Russia \\ ${ }^{3}$ Belgorod State Agricultural University, Russia \\ ${ }^{4}$ Kursk State Agricultural Academy, Russia
}

\begin{abstract}
This scientific article deals with the problem of antibiotic resistance. It is very difficult to give a complete picture of the resistance of microorganisms to antibiotics, because this topic is very diverse and is being actively investigated. Accordingly, information about more and more cases of antibiotic resistance appears very quickly. Less than a century has passed since the beginning of large-scale use of antibiotics. In this short historical period of time, the threat of antibiotic resistance has reached a global level, and it would be wrong to deny that it is humanity that has created such an enemy through its own efforts. Antibiotic resistance is a property of a microorganism that is associated with a decrease in the quality of the effect of an antibiotic on a given culture. The driving force behind this phenomenon is evolution. With the help of random mutations, an individual appears that is not susceptible to the effects of a previously used drug. The emergence of superbugs-cultures that do not respond to the use of currently existing antibiotics will lead to a decrease in the quality of life of people. Diseases that no longer caused concern in modern society will once again become deadly.
\end{abstract}

\section{Introduction}

In our rapidly changing world, in the age of modern computer technologies, joint enterprises and companies, international projects, it is necessary to be able to adopt foreign experience in various areas of human life [1. - p. 230-234].

International consolidation of scientific knowledge in the field of medical ecology aimed at public health is one of the priority tasks in the world [2. - p. 111].

Currently, when the use of antibiotics is widespread, resistant forms of microorganisms are very common. Resistance to one degree or another in most bacteria is observed in almost all antibiotics used. The ability of microbes to form resistant forms significantly reduces the effectiveness of antibiotics, especially in medicine.

The principle of action of antibiotics is based on an obstacle to the normal functioning of the bacterial cell: a violation of the synthesis of the cell wall, the permeability of the cell membrane, intracellular protein synthesis, the synthesis of nucleic acids and folic acid. The negative effect of antibiotics on bacteria contributes to the development of mutations in microorganisms, as a result of which the latter may develop resistance to the action of drugs, the so-called "antibiotic resistance". This is also due to the fact that bacteria are able to exchange information (intraspecific and interspecific), and multiply rapidly, which contributes to a rapid and productive response to changed adverse environmental conditions. Those organisms that mutated in the right direction in a timely manner become antibiotic-resistant and continue to live and multiply in the host body [3. p. 431-433].

Antibiotic resistance is a natural process by which bacteria develop resistance to the drugs used to control them over time. As resistance develops, the effectiveness of drugs gradually decreases and eventually is lost completely. Antibiotic resistance is a consequence of their use, and the irrational use of antibiotics accelerates its occurrence (Carre B. et. al. 2012).

Irrational use of antibiotics is the use of them unnecessarily, for a too short period of time, in too small doses, taking drugs of inadequate effectiveness, or against a disease that this drug does not treat. According to the results of the assessment of outpatient records, 25 $\%$ of prescriptions did not correspond to the expediency of using these drugs, and $18 \%$ of prescriptions did not have documentary indications. Most often, sulfonamide and other anti-infective agents were used without documentation [4. - p. 201-203].

In the United States, antibiotics are most commonly used on an outpatient basis, and $30 \%$ to $50 \%$ of prescriptions may be inappropriate [5 - pp. 411-415].

The frequency of medical errors when using penicillin group antibacterial drugs was $36.9 \%$ of prescriptions. At the same time, in $17 \%$ of these cases, two or more errors were made simultaneously in the treatment of the patient. A third of medical errors (32.9 $\%)$ are caused by various violations of the dosage regimen. $29.8 \%$ of all cases of incorrect use of the drug are associated with the appointment of an antibiotic in the absence of indications or for an unregistered indication. $13.0 \%$ of the identified medical errors relate

\footnotetext{
Corresponding author: adiosamigo1995@yandex.ru
} 
to the use of an antibacterial drug in the presence of contraindications to its use. Most often (in 92\% of cases) such a contraindication was the presence in the past of the patient's allergy to this drug or to other beta-lactam antibiotics [6. - p. 511-521].

Self-medication is defined by the World Health Organization as the main factor leading to excessive use, misuse of antibiotics and resistance to them [7. - p. 329331].

The study in Novi Sad, Serbia, consisted of an inventory of all medicines in families and a survey on the practice of using these drugs. The main indicator was the number of antibiotics obtained without a prescription. Of the 112 families surveyed, 55 (49.1\%) had antibiotics. Antibiotics accounted for $11.98 \%$ $(92 / 768)$ of the total number of medicines in households. Of all the antibiotics in 41 families (44.57\%), they were not used at the present time and represent the remnants of previous treatment [8.- p. 109]. Antibiotics were usually purchased by prescription $(67,67.7 \%)$, while about a quarter of the packages were used for selfmedication, bought at a pharmacy without a prescription $(19,20,65 \%)$ or received from friends or family members $(6,6.52 \%)$ [9. - Pp. 507-513].

A study of older residents in Lebanon found that 61 $\%$ of respondents believed that antibiotics should be taken for colds, and $83 \%$ knew that improper use of antibiotics could lead to microbial resistance. Selfmedication significantly correlated with a lower level of education $(\mathrm{P}=0.036)$. Those who did not know about antibiotics stopped taking antibiotics at the wrong time $(P=0.002)$. The socio-economic status, gender, and age did not correlate with self-medication [10. - p. 384-388]. Against the background of economic difficulties, many patients stop taking antibiotics as soon as they feel better, which can happen before the microbes have been completely eliminated (Kazachkova N. M. 2017).

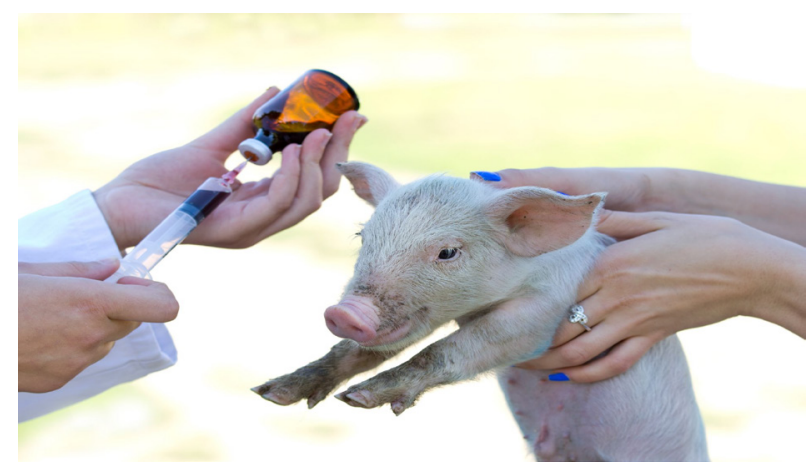

Fig. 1. The use of antibiotics in veterinary medicine.

The irrational use of antibiotics puts the body in great danger.

The problem of antibiotic resistance may be as follows: the effect of taking a course of antibiotics may be insignificant or completely absent, in this regard, the disease can progress and give complications, the greatest danger is in cases where there is a defeat of vital organs by a bacterial infection.

Inappropriate use of antibiotics is undoubtedly part of the overall problem, but antibiotic resistance is much broader. Since antibiotic resistance is a natural process that all microorganisms can eventually develop to the drugs used to fight them. However, through the implementation of national strategies and the rational use of medicines by health professionals and patients, as well as the appropriate use of antibiotics in veterinary and animal husbandry, combined with infection control, the development and spread of antimicrobial resistance can be significantly delayed.

In veterinary medicine, the use of antibiotics began immediately after their discovery.

Sick animals also need to be treated, but, as with humans, this treatment must be rational. The usual use of antibiotics on a large number of healthy animals, for example, to stimulate growth, is likely to lead to the emergence and spread of bacteria, resistant infections, both in humans and in animals. Resistant microorganisms carried by farm animals can be transmitted to humans through contaminated food, direct contact with animals, or through the environment, such as contaminated water.

\section{Materials and methods}

Work was carried out with domestic and foreign publications that provide data on factors affecting the formation of antibiotic resistance from various fields of medicine and veterinary medicine, on such resources as "pubmed", "eLibrary". The purpose of the work is to study the process of formation of antibiotic resistance and to develop basic recommendations for public education.

\section{Results and discussion}

Recently, antibiotic resistance has become the number one problem in the pharmacological industry engaged in the development of antimicrobial drugs. The fact is that it is characteristic of almost all known varieties of bacteria, in connection with which antibiotic therapy is becoming less effective. Common pathogenic microorganisms such as staphylococci, Escherichia coli, and Pseudomonas aeruginosa, proteas have resistant strains that are more common than their antibioticexposed ancestors are.

There is a distinction between natural and acquired resistance to antibiotics. With natural (primary) resistance, the microorganism does not have structures that the drug acts on, or produces enzymes that inactivate it. Acquired (secondary) resistance occurs as a result of contact of the microorganism with an antimicrobial agent. Numerous mechanisms of antibiotic resistance development are known. In bacteria, the targets for drugs are modified, the permeability of their external structures changes, and shunts are formed, which provide a rapid release of the antibiotic from the microbial cell. Enzymes that inactivate the antibiotic (penicillinases, cephalosporinases, beta-lactamases, levomycetin acetyltransferases, etc.) are often induced. The danger lies in the wide and rapid spread of acquired resistance 
between microorganisms. The drugs become ineffective even in patients who have never used them.

Resistance to different groups of antibiotics, and even to individual drugs, develops in different ways. Long-known penicillins and tetracyclines, as well as newer developments in the form of cephalosporins and aminoglycosides, are characterized by a slow development of antibiotic resistance, and their therapeutic effect decreases in parallel with these. What can not be said about such drugs is that the active substance is streptomycin, erythromycin, rimfampicin and lincomycin. Resistance to these drugs is developing rapidly, and therefore the appointment has to be changed even during the course of treatment, without waiting for its end. The same applies to the drugs oleandomycin and fusidine.

All this suggests that the mechanisms of development of antibiotic resistance to different drugs differ significantly [11. - p. 22-25].

A large number of errors that lead to the appearance of antibiotic-resistant strains of superbacteria appear even at the stage of forming a treatment regimen for a certain patient. At the same time, the remnants of the previous prescribed course of antibiotics are used by the population for self-treatment, and are used in cases where the use of these drugs is unjustified.

The use of antibiotics in animal husbandry poses potential public health risks, as their frequent and careless use can lead to the emergence of pathogenic bacteria that are resistant to commonly used antibiotics. Therefore, infectionswithresistantbacteriaaredifficulttotreat.

Resistant bacteria can be transmitted not only from person to person, but also through animals, food, and the environment. A well-known drug-resistant bacterium that occurs in both humans and animals is the MRSA bacterium.

The World Health Organization (WHO) in 1969 recommended that antibiotics used in public health should not be added to feed. Based on many years of experience with the mass use of antibiotics to stimulate the growth of productive animals, it was found that when using the same antibiotic in the agricultural complex for a long time, the effectiveness of the drug decreases sharply. At the same time, antibiotic-resistant strains of microorganisms accumulate in the gastrointestinal tract of the animal. It was found that the resistance of Escherichia coli increased to the antibiotic used when this drug is added to the feed. Since January 1, 2006, EU countries have officially banned the use of so-called feed antibiotics. However, the consequences of their mass use are still felt. For example, in the Netherlands, sales of veterinary antibiotics have been declining for many years. Since 2007, the indicator has decreased by more than $70 \%$. The number of resistant bacteria was also significantly reduced. About a decade ago, the Netherlands was a major consumer of antibiotics in the livestock sector. In 2008, a special group was established to work, inter alia, with representatives of the feed industry, veterinarians and representatives of the livestock sector. And, as the figures show, the work was successful.

It is not yet clear whether similar programs will be implemented in other countries. Perhaps this only applies to Europe. For many developing countries outside the European region, the solution still seems too far away and ambiguous. However, reducing the volume of antibiotics in the field of animal husbandry could be the first step towards taking care of the health of the population.

In Russia, the use of antibiotics is regulated, and a list of permitted antibiotics in animal husbandry and poultry farming is established. For the treatment and prevention of diseases of poultry and meat breeds of livestock, antibiotics are used, which are controlled without fail: chloramphenicol(levomycetin), tetracycline, streptomycin, penicillin, grisin, bacitrocin. Moreover, grisin and bacitrocin are not used in medicine $[1,8]$. In accordance with the instructions, treatment should not be carried out ten days before the slaughter of poultry, meat breeds of livestock - this period is necessary for the removal of antibiotics from the body of animals (for some antibiotics, a longer period is required). The producer of agricultural products is obliged to declare the use of antibiotics in the cultivation of animals during the sanitary and veterinary examination. If antibiotics were used and not declared, this is a violation of the law.

In Thailand, as a result of the implementation of the "Prudent Use of Antibiotics" program, the number of prescribed antibiotics decreased by $18-46 \%$. In Vietnam, a program was carried out, the essence of which was to inspect pharmacy employees for compliance with the rules for the sale of prescription drugs and to train employees in the principles of pharmacological treatment of major diseases, which significantly reduced the volume of sales of antibiotics for the treatment of ARVI. In 2010. The Medical University of Zambia has revised the curriculum for students to include topics on the resistance of microorganisms to antimicrobial drugs and the rational use of medicines [12. - p. 54-59].

To detect antibiotic resistance, there are phenotypic (traditional) and molecular genetic methods. Phenotypic methods include: the diffusion method (disco-diffusion), the method of serial dilutions (in agar, in broth) and the combined E-test. Despite the widespread use and introduction of modern automatic analyzers, phenotypic methods are associated with significant methodological difficulties. In accordance with MUC 4.2.1890-04 "Determination of the sensitivity of microorganisms to antibacterial drugs", when using phenotypic methods, the following requirements should be strictly observed. It is necessary to use antibiotic substances with a known level of activity, observe storage regimes, and carefully monitor the quality of nutrient media. In this regard, molecular genetic methods of studying antibiotic resistance can be used as a method of choice, especially in the following situations: studies of outbreaks of acute and chronic infections associated with the provision of medical care; verification of the results of phenotypic methods of intraspecific typing (antibiotic resistance) aimed at identifying hospital strains; when monitoring the circulation of international epidemic clones within 
territorial units, subjects of the Russian Federation and at the national level. Molecular genetic methods are aimed at identifying genes associated with resistance, and are characterized by: high sensitivity; speed of obtaining results; standardization and technological research. It is important that they do not require manipulations with live bacterial cultures, which helps to prevent the spread and circulation of microorganisms inside medical and diagnostic and laboratory facilities. Rapid diagnosis of infection is an important factor affecting the outcome of the disease: every hour of delay in effective antibacterial therapy for septic shock increases the risk of mortality by $7.6 \%$. Given the high level of antibiotic resistance and the wide distribution of carbapenemase-producing strains, the issue of rapid results is becoming urgent. Currently, non-cultural methods based on the principle of multiplex PCR, in situ fluorescence hybridization (FISH), etc. are becoming increasingly common to reduce the time of pathogen identification.

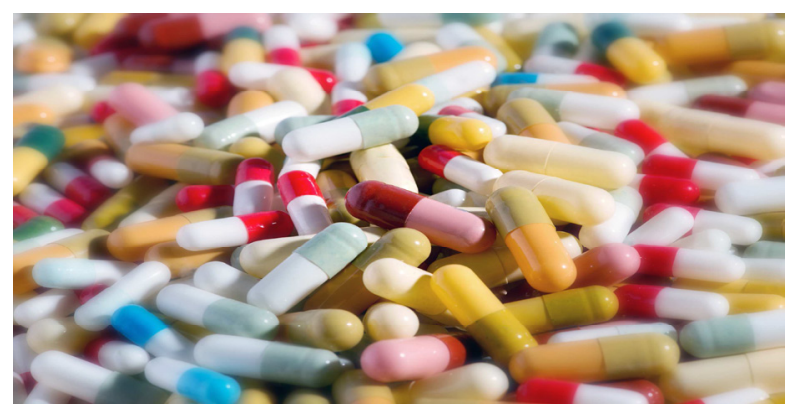

Fig. 2. Antibiotics.

The European Strategic Action Plan on Antibiotic Resistance identifies seven key areas for action to protect the health of Europeans. These areas are as follows:

- national multisectoral coordination of efforts to contain antibiotic resistance;

- monitoring of the use of antibiotics and their resistance;

- strategies for the rational use of antibiotics and increased surveillance of their use;

- infection control in medical institutions;

- emergence and spread of resistance to antibiotics used in veterinary medicine and agriculture;

- innovation and research on the development of new drugs and technologies;

- awareness, patient safety, and partnership.

Governments can take a leadership role by implementing comprehensive strategies to prevent the emergence of antibiotic resistance and ensuring that these strategies have an adequate resource base.

\section{Conclusion}

Antibiotic resistance is a serious threat to modern humanity. It is possible to slow down this process only by strengthening educational activities in different segments of the population. In medical organizations, it is necessary to provide additional educational material for employees, discuss clinical cases, and change established treatment protocols. Selling the exact amount of drugs needed for treatment can reduce the number of cases of self-medication. A better and more informed approach to the use of antibacterial drugs is needed.

In the Russian Federation, the attitude to the problem of antibiotic resistance is highly responsible, and the proof of this is the project "Map of Antimicrobial Resistance in Russia". Such large organizations as the Research Institute of Antimicrobial Chemotherapy (Smolensk), the Interregional Association of Microbiology and Antimicrobial Chemotherapy (Smolensk), and the Scientific and Methodological Center for Monitoring Antibiotic Resistance (Smolensk) are engaged in research in this area, collecting information and systematizing it to fill the antibiotic resistance map. Smolensk), created on the initiative of the Federal Agency for Health and Social Development. The information provided within the framework of the project is constantly updated and is available to all users who need data on antibiotic resistance and effective treatment of infectious diseases.

Agricultural workers can assist in the fight against antimicrobial resistance by improving the health of farm animals, ensuring proper sanitary conditions and compliance with agricultural regulations and regulations. Veterinarians should rationally prescribe antibiotics, especially those that are of primary importance in medicine.

\section{References}

1. T. P. Boldyreva Materials of the All-Russian Scientific and Practical conference: in 4 parts $\mathrm{p}$. 230-234.-(Kursk: Publishing of Kursk. state agricultural AK, 2006)

2. I. N. Medvedev, M. M. Naumov, B. D. Bezpartochnyj Medical ecology of the KMA region: monograph, (Moscow, 2004)

3. K. Y. Savchenko, Young scientist. 22 (312), pp. 431-433 (2020).

4. M.J. Ray, G.B. Tallman, D.T. Bearden, M.R. Elman, J.C. McGregor BMJ. 367, 16461 (2019) doi: 10.1136/bmj.16461.

5. B. Sagge, M. Lessire, T. H. Nguyen Proc. XIX World Poultry Congress, vol. 3, 411-415. (Amsterdam, 2012)

6. T.A. Rowe, J.A. Linder Expert Rev Anti Infect Ther. 17(7), 511-521 (2019) doi: 10.1080/14787210.2019.1635455

7. A.V. Kuzmina, V. A. Polivanov, I. L. Asetskaya, S. K. Zyryanov KMAKH, 2, (2016)

8. A. Tomas, M. PautKusturica, Z. Tomić, O. Horvat, D. DjurovićKoprivica, D. Bukumirić, A. Sabo Int J Clin Pharm. 39(3), 507-513 (2017) doi: 10.1007/s11096-017-0461-3.

9. A. Jamhour, A. El-Kheir, P. Salameh, P.A. Hanna, H. Mansour Am J Infect Control. 45(4), 384-388. (2017) doi: 10.1016/j.ajic.2016.11.026. 
10. N. M. Kazachkova, S. R. Ishbulatova, G. K. Duskaev International Student Scientific Bulletin, 4-3, (2017)

11. E. Katzman Antibiotic resistance of microorganisms: methods of determination Retrieved from: http:// yandex.ru/turbo/ilive.com.ua/s/health/antibiotikor ezistentnost-mikroorganizmov-metodyopredeleniya_124530i15955.html

12. I. R. Kulmagambetov, S. S. Sarsenbayeva, Sh. Kh. Ramazanova, N. K. Yessimova International Journal of Applied and Fundamental Research. 9-1, 54-59 (2015) 